\title{
Effect of downstream cylinder rotation on wake dynamics of two inline circular cylinders
}

\author{
M NEERAJ PAUL and SHALIGRAM TIWARI* \\ Department of Mechanical Engineering, Indian Institute of Technology Madras, Chennai 600036, India \\ e-mail: neeri.89@gmail.com; shaligt@iitm.ac.in
}

MS received 17 March 2017; revised 1 November 2017; accepted 6 July 2018; published online 17 December 2018

\begin{abstract}
Two-dimensional numerical investigations have been carried out to study flow past two inline circular cylinders with stationary upstream and rotating downstream cylinder. Computations are performed using commercial software ANSYS Fluent 16.0 for a fixed Reynolds number (Re) of 150 to study wake characteristics unsteady laminar flow. Optimal values of spacing between cylinders and downstream cylinder rotation speed have been identified, confirming the suitability of such an arrangement for drag reduction and flow control. Steady and unsteady features of the wake have been examined with the help of vorticity contours, lift and drag coefficients, their signals and spectra. Interesting phenomena that include multiple transitions in the temporal behavior of the flow and appearance of circulation zone, its development, stabilization, and transformation have been elaborated in this study.
\end{abstract}

Keywords. Two inline circular cylinders; rotating downstream cylinder; inter-cylinder spacing; wake transition; circulation zone; lift and drag; flow control.

\section{Introduction}

Flow past circular cylinder has been a subject to large number of experimental and numerical investigations and forms a bench-mark problem in fluid flow studies. These studies reveal transitions in characteristics of the flow that arise due to interaction between viscous and inertial forces. Such interactions could cause vortex shedding triggered by shear layer instability and capable of inducing vibrations in neighboring structures. Such a phenomenon that results in compromise of structural integrity is referred to as 'vortex induced vibration' (VIV). Flow control to suppress vortex shedding is commonly achieved by both active as well as passive means.

Rotational motion imparted to a circular cylinder creates flow stabilizing effect in particular ranges of rotational speed which perhaps forms the main reason for availability of exhaustive literature on flow past rotating circular cylinders. The rotational motion results into redistribution of stagnation points and the stabilizing effect appears in the form of suppression of vortex shedding. The two decisive parameters that determine flow behavior in such cases are Reynolds number (Re) and non-dimensional rotational speed $(\alpha)$. The latter is defined as ratio of surface speed of cylinder to the mean speed of incoming flow and is expressed as $\alpha=\omega d / 2 U_{\infty}$, where $\mathrm{d}$ is cylinder diameter, $\omega$ is the

*For correspondence rotational speed and $U_{\infty}$ is the mean speed of incoming flow. For $\operatorname{Re} \leq 20$ and $\alpha \leq 0.5$, the flow exhibits a steady behavior as reported by Ingham [1]. Tang and Ingham [2] carried out numerical study on steady flow past rotating cylinders for two different values of Re, viz. 60 and 100 , and $\alpha$ in the range from 0 to 1 . However, for larger values of $\mathrm{Re}$, unsteadiness appears in the form of vortex shedding.

The behavior of unsteady flow past a rotating cylinder has been explored by Badr et al [3], Kang et al and Nair et al [5]. They report that the flow which is initially unsteady at $\operatorname{Re}>50$ for a stationary cylinder remains so for lower values of $\alpha$. However, when $\alpha$ is increased further, a critical value is reached beyond which the flow becomes steady with arrest of vortex shedding. Kang et al [4] have also presented a logarithmic relation between the critical rotational speed and Re. Their work describes a bifurcation curve that separates the stable and time periodic flow for an $\alpha$ range of $0-2$ and Re range of 40-160. Mittal and Kumar [6] further extended the range of $\alpha$ in their numerical study for $\mathrm{Re}=200$. Their work is the first to disclose existence of a second band of time periodic flow within $4.34<\alpha<4.70$. Experimental results of Kumar et al [7] confirm the existence of vortex shedding in this narrow band of rotational speed. The shedding frequency in this range with vortices of fixed sense of rotation is found to be nearly 8 times smaller than that observed at lower rotational speeds. Recently, Meena et al [8] and Rao et al [9] studied threedimensional instabilities in flow past a rotating circular 
cylinder for the same range of rotational speed. They have reported new modes of instabilities along the axial direction of cylinder that are dependent on its length. Even though both the studies consider three-dimensional effects, they reported that parallel time dependent vortex shedding (twodimensional) still remains the dominant mode of instability. Present study banks on this understanding while performing two-dimensional numerical investigations. Moreover, the choice of $\mathrm{Re}=150$ is based on the observations of Carmo and Meneghini [10] which confirm that for flow past two stationary cylinders in tandem the onset of three-dimensionality takes place only beyond $\mathrm{Re}=190$.

Apart from single cylinder rotation, arrangement of multiple cylinders may also exhibit suppression of vortex shedding. Flow past side by side arrangement of two counter rotating circular cylinders has been found to stabilize the flow beyond a critical speed of rotation dependent on inter-cylinder spacing. Recent studies that have dealt with this problem include [11-15]. As compared to flow past side by side arrangement of rotating cylinders, comprehensive study on flow past tandem rotating cylinders is limited in literature. Shaafi and Vengadesan [16] have investigated two-dimensional flow past rotating upstream control rod by varying the inter-cylinder spacing, blockage ratio and rotational speed. Ayyappan and Vengadesan [17] investigated the influence of placing a rotating control rod at staggering angles with respect to a stationary circular cylinder for two different values of control rod diameter and fixed rotational speed. Nemati et al [18] studied flow and heat transfer for flow past two rotating circular cylinders in tandem using Lattice Boltzmann method for selected values of spacing at $\mathrm{Re}=100$. Their work clearly suggests that further investigations need to be carried out on flow control aspect of the problem.

Mittal [19] carried out numerical study on flow past an arrangement of two small rotating control rods placed on either side of a large stationary circular cylinder. He reported that at small separations, there exists a critical rotational speed beyond which the flow becomes stable. On the other hand, Mittal and Raghuvanshi [20] considered placement of a small stationary control rod behind the main cylinder at different staggered locations. They reported that for low values of Re, an optimum positioning of the control rod can suppress vortex shedding. Present work investigates the effect of rotation of downstream circular cylinder on possibility of suppressing vortex shedding in the wakes of two inline cylinders.

\section{Problem definition}

The computational domain used in the present numerical study of flow past two circular cylinders with rotating downstream cylinder has been shown in figure 1 . The upstream cylinder is located at a distance of $15 \mathrm{~d}(\mathrm{~d}=\mathrm{di}$ ameter of either cylinder) from inlet (marked as ' 1 ') while

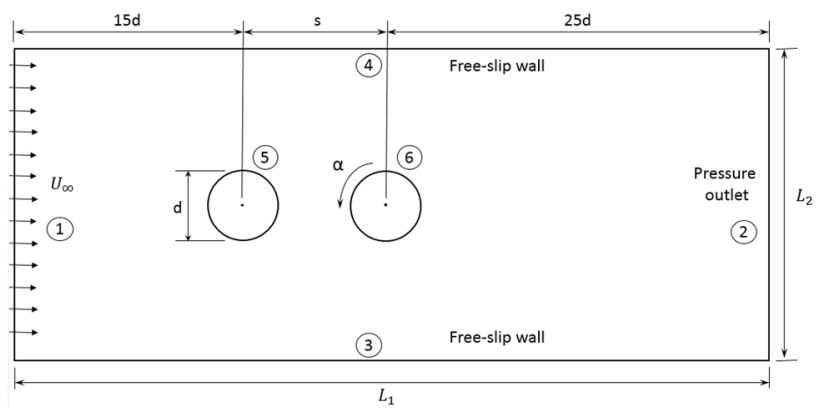

Figure 1. Computational domain.

the downstream cylinder is placed $25 \mathrm{~d}$ upstream from the outlet (marked as ' 2 '). The total length of the domain $\left(L_{1}\right)$ is $L_{1}=15 d+s+25 d$, where $s$ is the distance between centers of the two cylinders. The width of the domain $\left(L_{2}\right)$ is chosen as $25 \mathrm{~d}$ so as to minimize the effects caused by free-slip side wall. Computations are carried out for intercylinder spacing values of $1.5 \mathrm{~d}, 2 \mathrm{~d}, 3.5 \mathrm{~d}$ and $5 \mathrm{~d}$ while the non-dimensional rotational speed $\left(\alpha=\omega d / U_{\infty}\right)$ of the downstream cylinder is varied from 0 to 5.2. The Reynolds number $\left(\operatorname{Re}=\frac{\rho U_{\infty} d}{\mu}\right)$ is fixed equal to 150 for all the computations so that the flow remains unsteady and laminar while three-dimensional effects become negligible as reported by Carmo and Meneghini [10].

\section{Governing equation and boundary conditions}

The numerical solution to the considered problem has been obtained by solving the incompressible two-dimensional mass and momentum conservation equations that are given as

- Mass conservation

$$
\nabla \cdot \vec{u}=0
$$

- Momentum (Navier-Stokes) conservation

$$
\frac{\delta \vec{u}}{\delta t}+(\vec{u} \cdot \nabla) \vec{u}=-\frac{\nabla p}{\rho}+v \nabla^{2} \vec{u}
$$

where $\vec{u}$ represents the two-dimensional velocity vector in the Cartesian coordinate system for which $u$ and $v$ are the components along $x$ and $y$-directions, respectively. Here $p$, $\rho$ and $v$ represent the static pressure, density and kinematic viscosity, respectively. The boundary conditions imposed for the computational domain shown in figure 1 are listed below.

Face 1 (Inlet):

Uniform velocity; $u=U_{\infty}$ and $v=0$

Face 2 (Outlet):

Pressure outlet; $p=P_{\infty}$

Faces 3 and 4 (Side walls):

Free-slip and impermeable; $\frac{\partial u}{\partial y}=0, v=0$ 
Surfaces 5 and 6 (upstream and downstream cylinder surfaces):

Upstream cylinder (no-slip and impermeable); $u=0 v=0$

Downstream rotating cylinder (no-slip and impermeable): $u=u_{c}$ and $v=v_{c}$ where $u_{c}$ and $v_{c}$ are the Cartesian velocity components at the cylinder surface.

\section{Grid and numerical technique}

\subsection{Grid mesh}

The computational domain has been divided into structured quadrilaterals with the region close to cylinder having refined body-fitted mesh as shown in figure 2(a). The size of the quadrilaterals away from the cylinder increases in the ratio 1.12 as shown in figure 2(b). The cell aspect ratio for the entire domain is maintained below 4.0 in order to achieve a good quality mesh.

\subsection{Grid and domain independence tests}

Grid independence has been confirmed by varying the number of circumferential nodes $\left(N_{C}\right)$ around the cylinder surface. In the process, the radial divisions are modified so that the aspect ratio does not exceed 4.0. Figure 3 shows variations of coefficients of mean drag and lift on the cylinders for varying number of nodes on their surfaces. For considered values of $s=2 d$ and $\alpha=3$, the change in the values of force coefficients is found to be less than $1 \%$ when the grid is refined beyond $N_{C}=100$. Accordingly, $N_{C}=100$ has been chosen for all the computations.
A domain independence study was conducted for $\mathrm{s}=2 \mathrm{~d}$ and $\alpha=2.0$ to minimize the wall and end effects. The results presented in table 1 indicate that optimal distance of outlet from center of downstream cylinder $\left(\mathrm{L}_{\mathrm{co}}\right) \times L_{2}$ is $25 \times 25$.

\subsection{Numerical technique}

The governing equations are solved using finite volume based commercial solver ANSYS Fluent 16.0. The algorithm based on semi-implicit pressure linked equations (SIMPLE) has been employed for solution of mass and momentum conservation equations. The effect of surface velocity of the rotating downstream cylinder has been accounted for with the help of a User Defined Function (UDF). The flow behavior has been captured using implicit discretization for spatial derivatives and first order discretization for temporal derivative. The convective terms are discretized using second order upwind while the diffusive terms are discretized by central difference scheme. The fluxes at the cell faces are computed with the help of Least Square Cell-Based method.

\section{Results and discussions}

Computations have been carried out by varying angular rotations of the downstream cylinder for different values of inter-cylinder spacing. Both the parameters compete in influencing the flow characteristics while causing effects through proximity interference and angular momentum exchange. Effects of these parameters on flow characteristics reveal interesting transformations in the wakes behind (a)

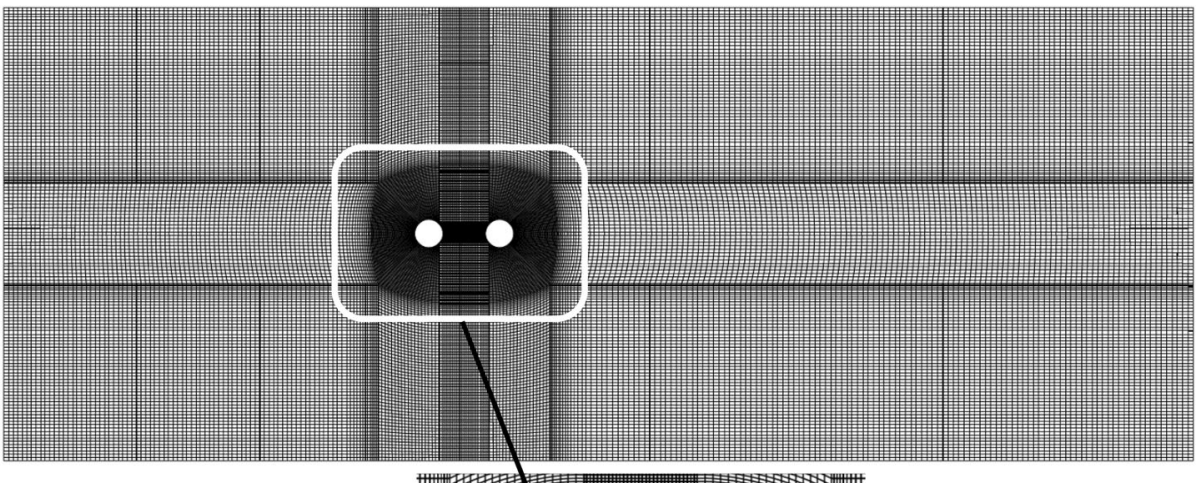

(b)

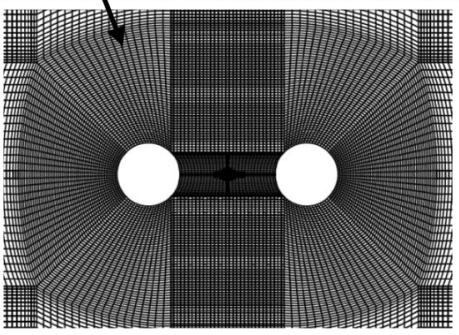

Figure 2. (a) Schematic of grid mesh in whole domain, (b) magnified view of mesh close to cylinder. 


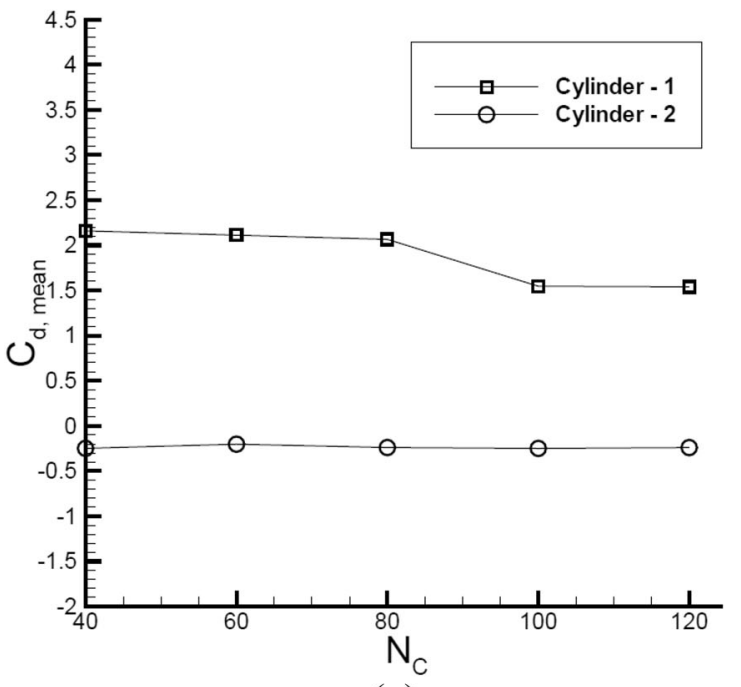

(a)

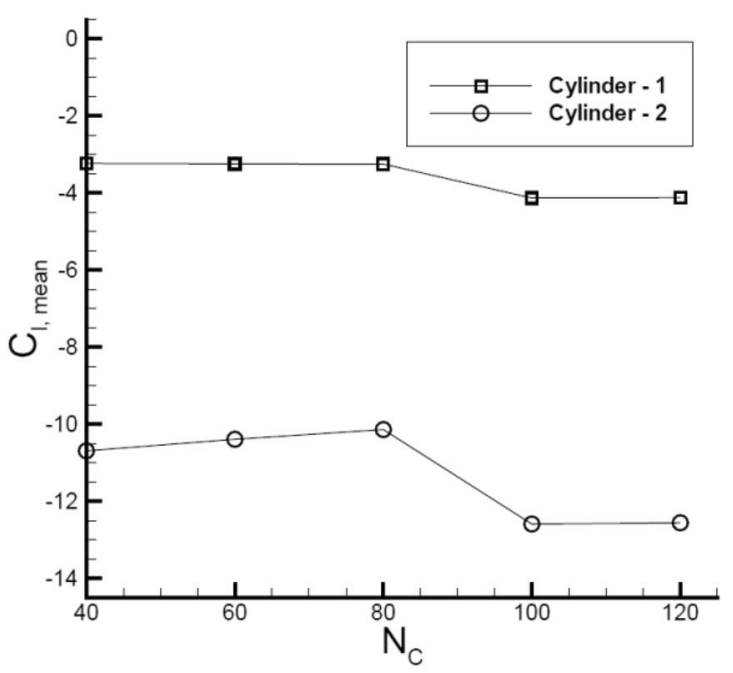

(b)

Figure 3. Variation of $C_{l}$ and $C_{d}$ for different grids for $\mathrm{s}=2 \mathrm{~d}$ and $\alpha=3$.

Table 1. Domain independence study for flow past tandem circular cylinder with downstream cylinder rotation for $\mathrm{s}=2 \mathrm{~d}$ and $\alpha=2.0$.

\begin{tabular}{lcc}
\hline $\begin{array}{l}L_{c o} \times \\
L_{2}\end{array}$ & $\begin{array}{c}\text { Strouhal } \\
\text { number }(\mathrm{St})\end{array}$ & $\begin{array}{c}\text { Mean drag coefficient on } \\
\text { downstream cylinder }\left(C_{d, 2}\right)\end{array}$ \\
\hline $20 \times 20$ & 0.07 & 9.821 \\
$20 \times 25$ & 0.072 & 9.635 \\
$25 \times 25$ & 0.073 & 9.428 \\
$25 \times 30$ & 0.073 & 9.403 \\
$30 \times 25$ & 0.073 & 9.427 \\
\hline
\end{tabular}

both the cylinders. Attempt has been made in the present study to explain the mechanism governing such transformations.

\subsection{Validation of computations}

The computational technique employed in the present study has been validated against the cases of flow past single rotating circular cylinder and two inline stationary cylinders reported in literature. Table 2 presents a comparison of mean force coefficients $\left(C_{l}\right.$ and $\left.C_{d}\right)$ and wake Strouhal number $(S t)$ for the case of flow past single rotating circular cylinder. Here, the non-dimensional rotational speed $(\alpha)$ has been fixed as 1.0 while two different values of $\operatorname{Re}=100$ and 200 are considered. The results obtained from the present computations show close match with those reoprted in literature. Similar conformity has been observed for flow past two inline stationary circular cylinders by considering variation of mean values $C_{d}$ for different values of $s$ as shown in figure 4. The subscripts ' 1 ' and ' 2 ' refer to upstream and downstream cylinders, respectively. The
Table 2. Validation of force coefficients for flow past a rotating circular cylinder.

\begin{tabular}{lccccc}
\hline & $\operatorname{Re}$ & $\alpha$ & $C_{\boldsymbol{l}}$ & $C_{\boldsymbol{d}}$ & $S t$ \\
\hline Kang et al [4] & 100 & 1 & -2.483 & 1.097 & 0.165 \\
Present work & 100 & 1 & -2.485 & 1.101 & 0.165 \\
Kumar et al [7] & 200 & 1 & - & - & 0.195 \\
Mittal and Kumar [6] & 200 & 1 & -2.375 & 1.09 & 0.190 \\
Present work & 200 & 1 & -2.385 & 1.104 & 0.192 \\
\hline
\end{tabular}

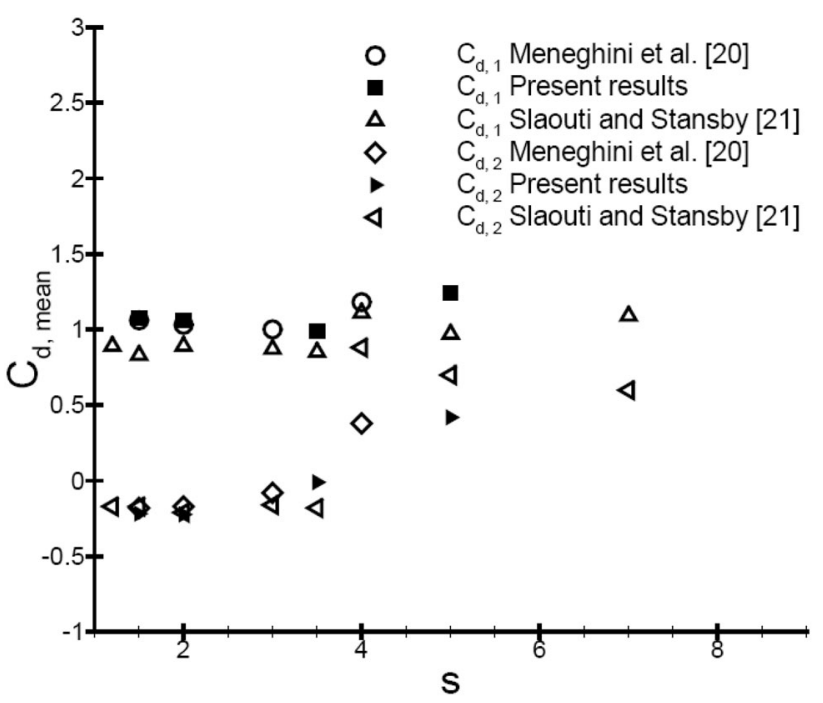

Figure 4. Variation of mean drag coefficient with inter-cylinder spacing for flow past two stationary circular cylinders in tandem.

scatter clearly shows that the drag coefficients on inline cylinders are in close match with the numerical results presented by Meneghini et al [21] obtained using Fractional 
step method and those by Slaouti and Stansby [22] using Random vortex method.

Figure 5 shows vorticity contours for different values of inter-cylinder spacing (s) for flow past two stationary cylinders in tandem. For a spacing of $1.5 \mathrm{~d}$, both cylinders behave like a single body which is confirmed by the absence of vortex shedding behind upstream cylinder and reattachment of shear layer onto the downstream cylinder. The same could be said for $\mathrm{s}=2 \mathrm{~d}$ but here the vortices are pinched-off further downstream as compared to the previous case. When the spacing is increased further, say $\mathrm{s}=2.5 \mathrm{~d}$ or $3 \mathrm{~d}$, there is weak interaction between the shear layers in the space between the cylinders. This causes asymmetry in the vortical region between the cylinders and induces instability due to which the shear layers attach alternately onto the downstream cylinder. These observations are in agreement with the experimental findings of Igarashi [23] wherein he calls the vortices as "quasi-stationary'. As the spacing is increased from $3 \mathrm{~d}$ to $3.5 \mathrm{~d}$, vortex shedding takes place closer to the downstream cylinder and the inter-cylinder region becomes more unstable. For a spacing of 5d, geometrically contrasting vortices (differ in shape and orientation) are shed from each of the cylinders. The vortices shed from the upstream cylinder appear to have shape and orientation similar to those observed at smaller spacing, whereas the vortices shed from the downstream cylinder appear inter-connected and oriented in longitudinal direction giving the appearance of 'braided hair'. A closer look reveals the presence of a weak elongated clockwise vortex below the counter-clockwise vortex which gets quickly dissipated as the flow progresses. It appears to be torn from the clockwise vortex that rolls over the top surface of the downstream cylinder confirming orientation of impinging vortex to be the main cause for such flow structure. These observations confirm the inferences drawn by Meneghini et al [21] wherein they suggest that the mechanism stimulating vortex generation behind the downstream cylinder gets triggered by fluctuations in the impinging flow.

Above results clearly establish the influence of proximity interference on wake flow behavior. At small separation ( $\mathrm{s}=1.5 \mathrm{~d}$ ), the cylinders act as a single body trapping a pair of standing vortices in the space between them. For large separation $(\mathrm{s}=5 \mathrm{~d})$, instability grows in the shear layers of the upstream cylinder resulting in vortex shedding behind both the cylinders. Based on these observations, three representative values of inter-cylinder spacing, viz., $1.5 \mathrm{~d}$, $3.5 \mathrm{~d}$ and $5 \mathrm{~d}$ have been considered which are classified as small, medium and large.

\subsection{Flow field characteristics}

5.2a Small spacing: Figure 6 shows the vorticity contours corresponding to an instantaneous flow field for $\mathrm{s}=1.5 \mathrm{~d}$ and $\alpha$ in the range from 0 to 5.2. The flow is unsteady up to $\alpha=1$, tends to get stabilized near $\alpha=1.5$ and again becomes unsteady around $\alpha=2$. As the value of $\alpha$ is increased further, another pair of steady-unsteady transitions appear for $\alpha$ values of 2.5 and 3.6, respectively. For

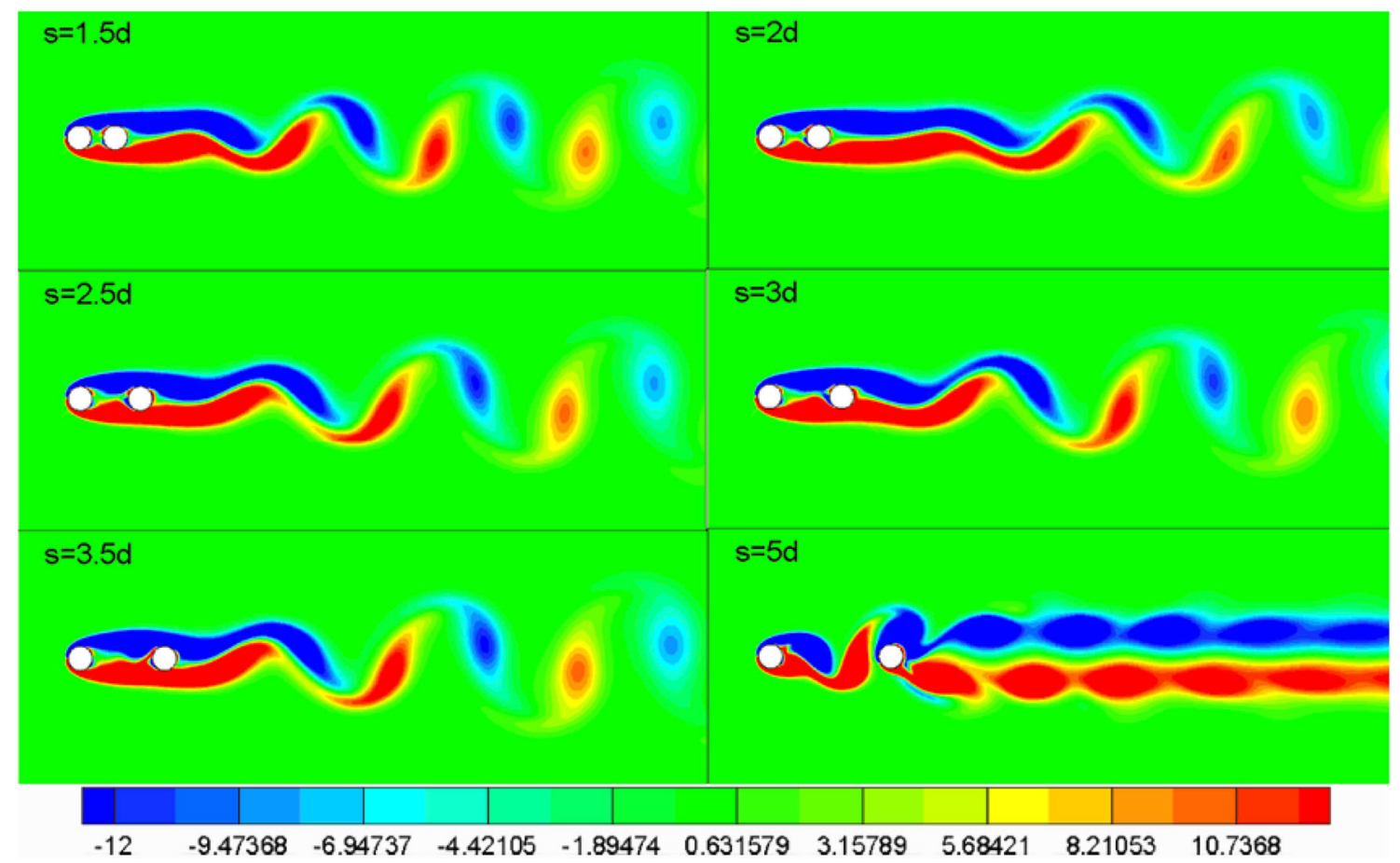

Figure 5. Instantaneous vorticity contours for different values of $\mathrm{s}$ at $\alpha=0$. 


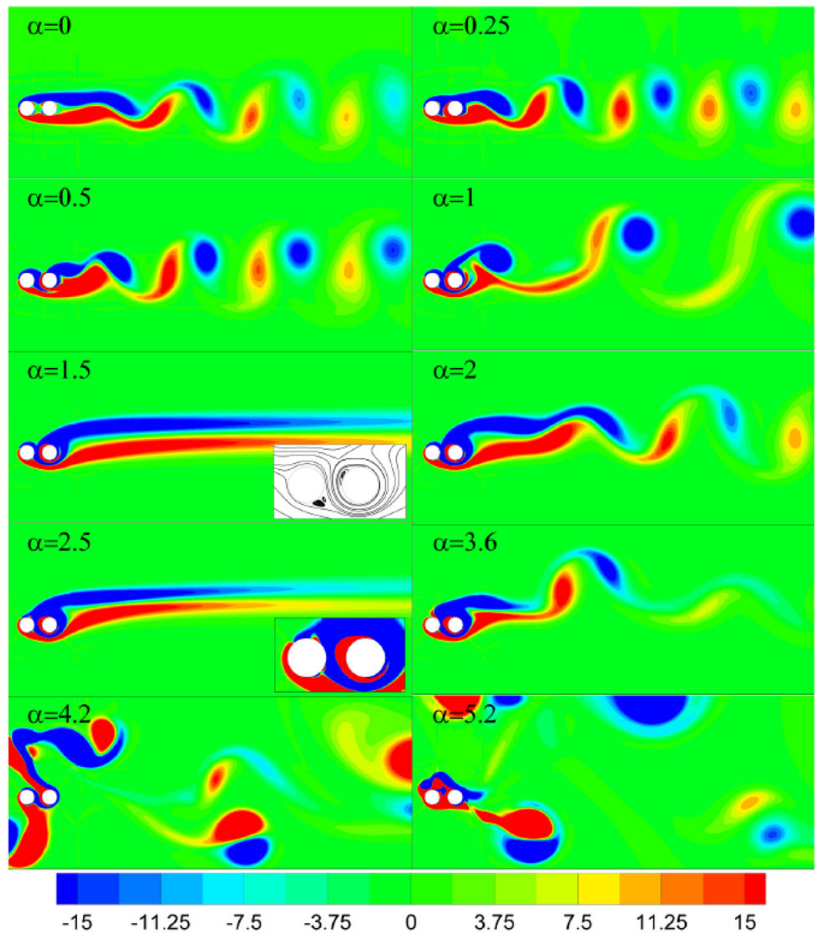

Figure 6. Instantaneous vorticity contours for different values of $\alpha$ and $\mathrm{s}=1.5 \mathrm{~d}$.

larger values of $\alpha=4.2$ and 5.2, non-periodic vortices are observed that are capable of disturbing even the upstream flow. It is to be noted that vortices are shed from the downstream cylinder alone for the considered range of rotational speeds. As $\alpha$ is increased from 0 to 1 , the strength of clockwise vortices is observed to increase whereas the counter-clockwise vortices get weakened due to stretching and elongation. Figure 6 clearly shows that the overall flow turns towards positive y-direction and loses symmetry about the horizontal axis, an observation similar to the case of flow past single rotating cylinder as reported by Mittal and Kumar [6]. For $\alpha$ values of 0 and 0.25 , the shear layers from the top and bottom surfaces of both the cylinders merge together resulting in vortex shedding in the wake of downstream cylinder. However, when $\alpha=0.5$, the shear layer from the top surface of upstream cylinder gets dragged into the inter-cylinder space and annihilates the presence of shear layer from the bottom surface. Consequently, the shear layer from the bottom surface grows and wraps downstream cylinder with further increase in rotational speed. When $\alpha=1$, the shear layers from the top surfaces of both the cylinders again merge. However, unlike the cases of $\alpha=0$ and $\alpha=0.25$, a part of this shear layer continues to envelope the downstream cylinder. Such a merger of shear layers from top surfaces happens to be unstable, breaking and remerging and synchronous with the vortex shedding frequency. Mode of vortex shedding is also found to change from ' $2 S$ ' to ' $S+P$ ' as per the classification of Williamson and Roshko [24]. On further increasing the rotational speed, the shear layer completely wraps around the downstream cylinder and makes the flow stable as shown in the case of $\alpha=1.5$. It is also observed that for this value of $\alpha$, the shear layers stretch far downstream behind the cylinders. This observation is similar to that made by Mittal and Kumar [6] for the case of flow past a single rotating cylinder at higher rotational speed.

For $\alpha$ values lying between 1.5 and 3.6, a bulk of fluid circulates around the rotating cylinder creating a 'virtual body' which will be identified as 'circulation zone' in the later part of discussion. At $\alpha=2$, the wake resumes vortex shedding in the locations farther downstream which again gets arrested at $\alpha=2.5$. With increase in value of $\alpha$ from 1.5 to 2.5 , the circulation zone of rotating cylinder grows and approaches upstream cylinder. This growth obstructs the flow path and offers resistance to free stream flow. At $\alpha=2.5$, the circulation zone seems to occupy whole of inter-cylinder space and moves further upstream such that it becomes indistinguishable from the shear layer of the upstream cylinder. When $\alpha$ is further increased to 3.6, clockwise vortices are shed from the top surface of the upstream cylinder as the circulation zone approaches its forward stagnation zone. The mode of shedding is noted to be '3P'. This shedding appears due to imbalance caused by opposing effects of momentum exchange between free stream and cylinder rotation. On further increasing the rotational speed, the flow becomes non-periodic and vortices are released from surface of rotating cylinder in all directions as shown for $\alpha$ values of 4.2 and 5.2. Some of these vortices propagate even in the upstream direction which indicates that they have gained enough velocity to overcome the mean flow. When such vortices break free and move in-front of the upstream cylinder, entire flow gets disturbed paving way for its non-periodic characteristics.

5.2b Medium spacing: For an inter-cylinder spacing of 3.5d, figure 7 shows three distinguishable transitions within the chosen range of rotational speeds. The wake exhibits vortex shedding from $\alpha=0$ to $\alpha=1$ that is similar to the case of $\mathrm{s}=1.5 \mathrm{~d}$ but differs in structure of vortices. For $\alpha=0.25$, geometrically contrasting vortices are shed from the downstream cylinder with no shift in the upstream stagnation zone of the stationary cylinder, indicating that rotation has not yet influenced the overall flow. For $\alpha=0.5$, two clockwise as well as anticlockwise vortices are shed behind the downstream cylinder in one cycle of vortex shedding resulting in ' $2 \mathrm{~S}+\mathrm{P}$ ' mode of shedding.

On further increasing $\alpha$ to 1 , the vortices get transmitted in negative y-direction, contrary to that observed for lower values of inter-cylinder spacing. The vorticity contour shows that the anticlockwise vortex generated near top surface of the rotating cylinder gets pulled into the gap between the cylinders and is pushed out from the lower surface of the rotating cylinder. Meanwhile, a clockwise vortex gets shed from top surface of the downstream cylinder. As the anticlockwise vortex moves through the 


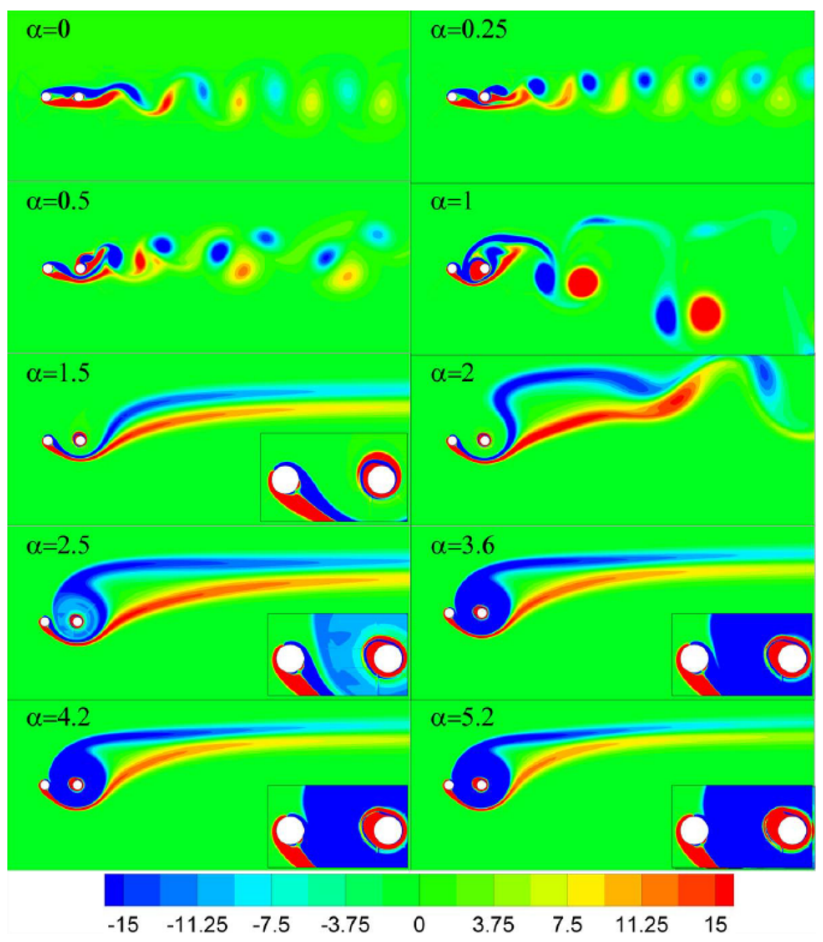

Figure 7. Instantaneous vorticity contours for different values of $\alpha$ and $\mathrm{s}=3.5 \mathrm{~d}$.

inter-cylinder space from the top surface to the bottom surface of the rotating cylinder, it gains net downward momentum making it move in negative y-direction. These observations are well confirmed with the help of temporal animations of wake flow and the mode of vortex shedding has been identified as ' $\mathrm{P}+\mathrm{S}$ '. For $\alpha=1.5$, vortex shedding is found to get arrested and the shear layers of the rotating cylinder wrap around one another forming in spiral while the shear layers of the upstream cylinder get dragged behind the downstream cylinder and extend further downstream. With further increase in $\alpha$, this extended shear layer becomes unstable causing vortex shedding far downstream as shown for case of $\alpha=2$. The flow again becomes stable when $\alpha$ approaches close to 2.5 and remains so for higher rotational speeds. The size of circulation zone keeps growing until it touches the upstream cylinder as seen for $\alpha=5.2$. For the same rotational speed, non-periodic shedding is observed for separation of $1.5 \mathrm{~d}$ as shown before. Such a difference in behavior is attributed to the size of circulation zone. A larger circulation zone would require greater rotational energy to evolve the flow to non-periodic mode observed for spacing of $1.5 \mathrm{~d}$.

5.2c Large spacing: For an inter-cylinder spacing of $5 \mathrm{~d}$ at lower rotation speeds, rotation of the downstream cylinder has little influence on the upstream cylinder as depicted in figure 8 for $\alpha<1$. Vortices are shed periodically from the upstream cylinder in contrast to small and medium spacing values.

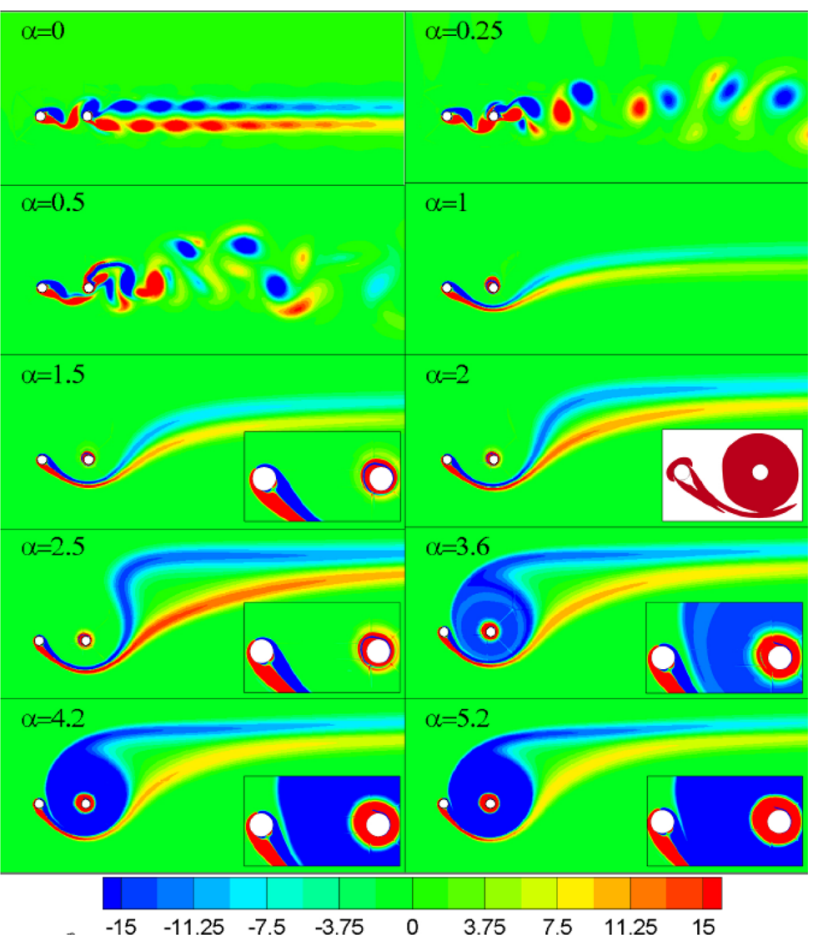

Figure 8. Instantaneous vorticity contours for different values of $\alpha$ and $\mathrm{s}=5 \mathrm{~d}$.

These vortices strike the rotating downstream cylinder and influence its wake as evident from vorticity contours corresponding to $\alpha$ values of 0.25 and 0.5 . However, when $\alpha=0.5$, the overall flow becomes non-periodic. This is due to interference between pulsating effect caused by shedding from upstream cylinder and the rotational effect of downstream cylinder. Similar to observations made for small and medium spacing, with further increase of $\alpha$, rotational effect becomes dominant causing arrest of vortex shedding. The velocity deficit region that grows wider downstream of a bluff body may be the cause for development of large circulation zones as shown in figure 8 . This region of slow moving fluid is easily influenced by the rotating cylinder and is dragged around creating a circulation zone that grows in size with increase in $\alpha$. For $\alpha$ in the range from 1 to 2.5 , the vorticity contour does not clearly represent distinguishable coherent structure in flow field (circulation zone) because vorticity of the circulation zone matches with that of free stream. To ascertain the presence of circulation zone, regions on flow domain having ' $Q$ ' value less than -1000 have been plotted in figure 8 for $\alpha=2$. The concept of Q-criterion introduced by Jeong and Hussain [25] gives local balance between shear strain rate and vorticity magnitude. It is expressed as $Q=0.5\left(\Omega^{2}-S^{2}\right)$, were $\Omega$ and $S$ represent magnitudes of local vorticity and strain rate tensors. Thus it becomes apparent from figure 8 that circulation zone could be characterized as region of large strain rate. 


\subsection{Development of circulation zone}

Streamlines in figure 9 illustrate development and growth of the circulation zone with increase in $\alpha$ for an inter-cylinder spacing of $3.5 \mathrm{~d}$. The first appearance of circulation zone is always associated with vortices being shed from upstream stagnation zone of the rotating cylinder as shown in figure 9(a), (b) and (c) at three different time instants for $\alpha=1$. The vortex generated on top surface of downstream cylinder is engulfed by the flow that circulates around this cylinder. It is then pulled through the inter-cylinder space breaking the circulation zone and gets dislodged in the wake of downstream cylinder. This vortex becomes an observable characteristic of circulation zone and may be referred to as 'vortex bubble'. The vortex bubble is created due to interaction between impinging free stream flow and circulating flow close to surface of the cylinder. These two counter-effects get equally matched for $\alpha=1$ thereby making the flow unstable. However, on further increasing $\alpha$, the effect of circulating flow becomes stronger thereby preventing the vortex bubble from getting dislodged. Moreover, the upstream stagnation zone separates from cylinder surface as shown in figure 9(d), (e) and (f). On increase of $\alpha$ from 1.25 to 2.5, the circulation zone grows in size dragging more fluid along its path accompanied with small increase in size of the vortex bubble.

Evolution in shape of the circulation zone and change in location of the stagnation point with increase in $\alpha$ has been presented in figure 10. The dotted closed curves represent stream lines that lie close to stagnation points. In each of figure 10(a), (b) and (c), only two stream lines have been shown for values of $\alpha$ corresponding to formation of a stable circulation zone $(\alpha=1.2)$ and the largest rotational speed $(\alpha=5)$. In similar way, for the stagnation points shown in each figure, other streamlines can be drawn. The results clearly illustrate a transformation in the shape of the stabilized circulation zone and the mechanism that leads to creation and growth of the circulation zone is almost alike irrespective of the spacing considered. With increase in $\alpha$ up to a particular value (highlighted in figure 10), the circulation zone grows in shape of a 'droplet' with the stagnation point acting as its apex. Beyond this value of $\alpha$, the circulation zone acquires an elliptic shape while approaching the upstream cylinder. Such transformation in shape of the circulation zone would influence the dynamic characteristics of flow.

\subsection{Dynamic characteristics}

5.4a Variation of drag: Variation of drag coefficients for both the cylinders with change in rotational speed has been presented in figure 11 for different values of inter-cylinder spacing. The mean drag coefficient on either cylinder is expressed as $C_{d}=C_{d, f}+C_{d, p}$, where $C_{d, f}$ refers to coefficient of skin friction drag and $C_{d, p}$ refers to pressure or form drag coefficient. The trend in variation of drag force on upstream cylinder is similar for all the spacing values considered except for the last four points corresponding to $\mathrm{s}=1.5 \mathrm{~d}$ shown in figure 11(a). These points coincide with

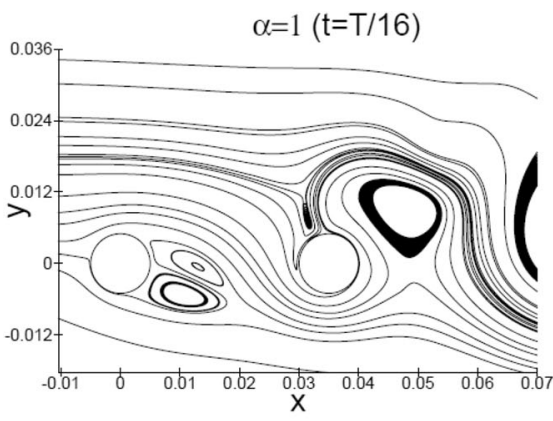

(a)

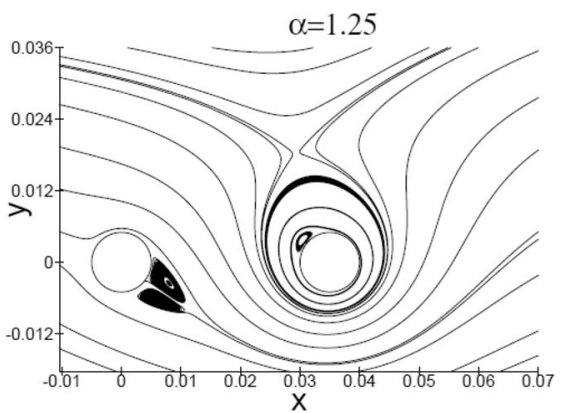

(d)

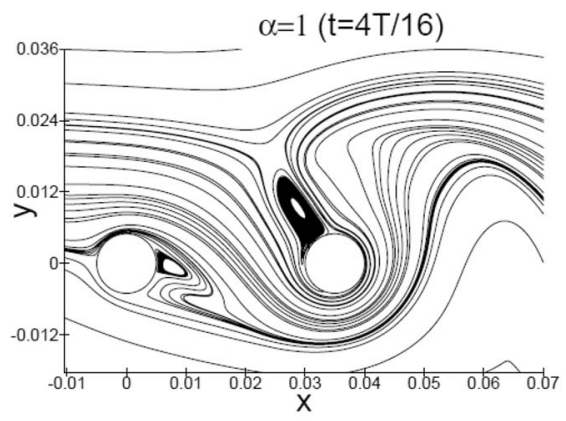

(b)

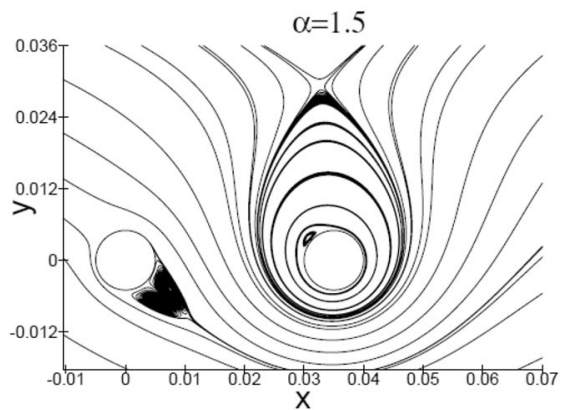

(e)

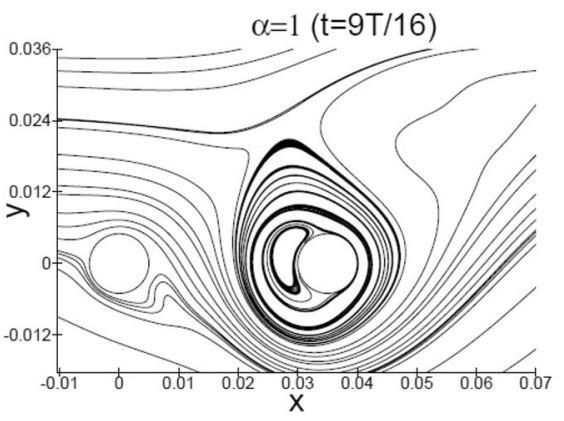

(c)

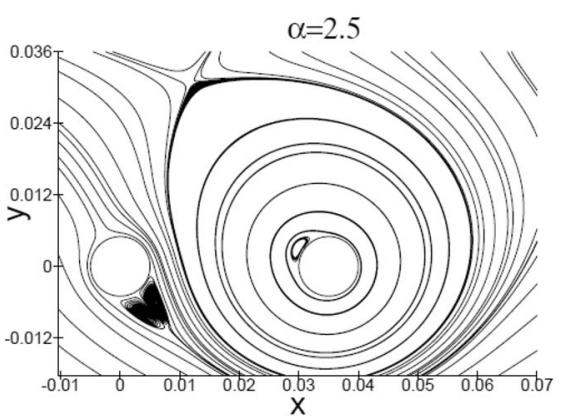

(f)

Figure 9. Streamline plots for $\mathrm{s}=3.5 \mathrm{~d}$ (a) $\alpha=1$ and $\mathrm{t}=\mathrm{T} / 16$, (b) $\alpha=1$ and $\mathrm{t}=\mathrm{T} / 4$, (c) $\alpha=1$ and $\mathrm{t}=3 \mathrm{~T} / 4$; steady streamlines for $(\mathbf{d})$ $\alpha=1.25,(\mathbf{e}) \alpha=1.5$, (f) $\alpha=2.5$. 


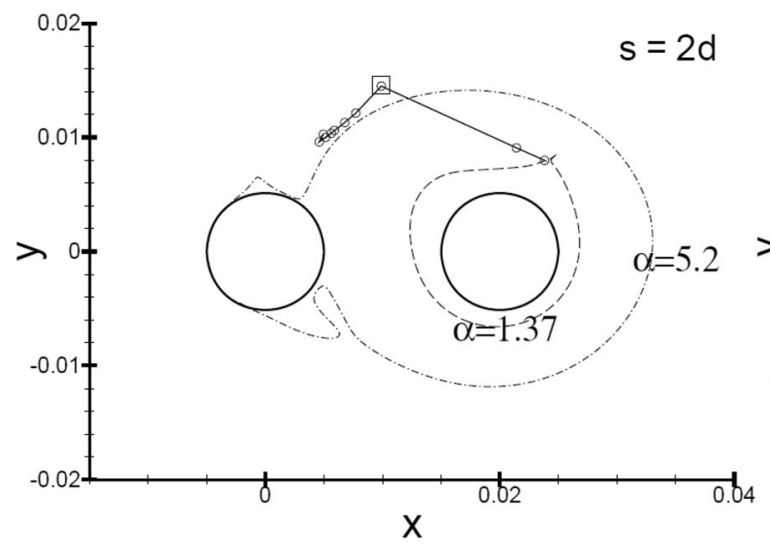

(a)

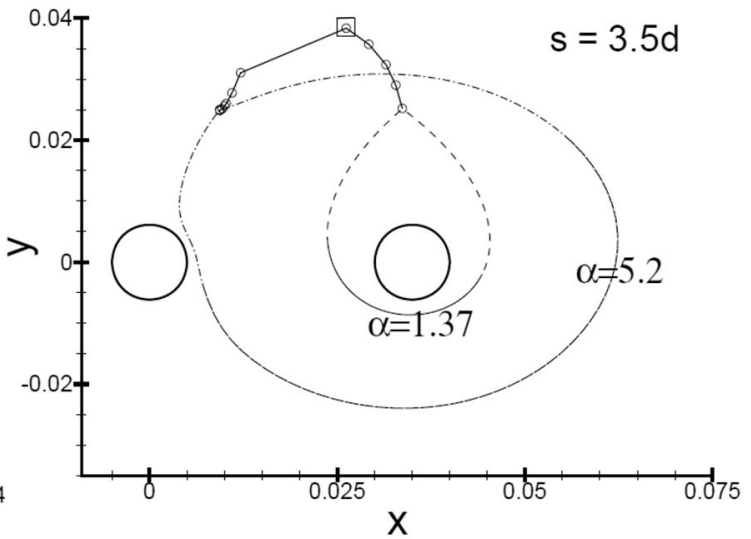

(b)

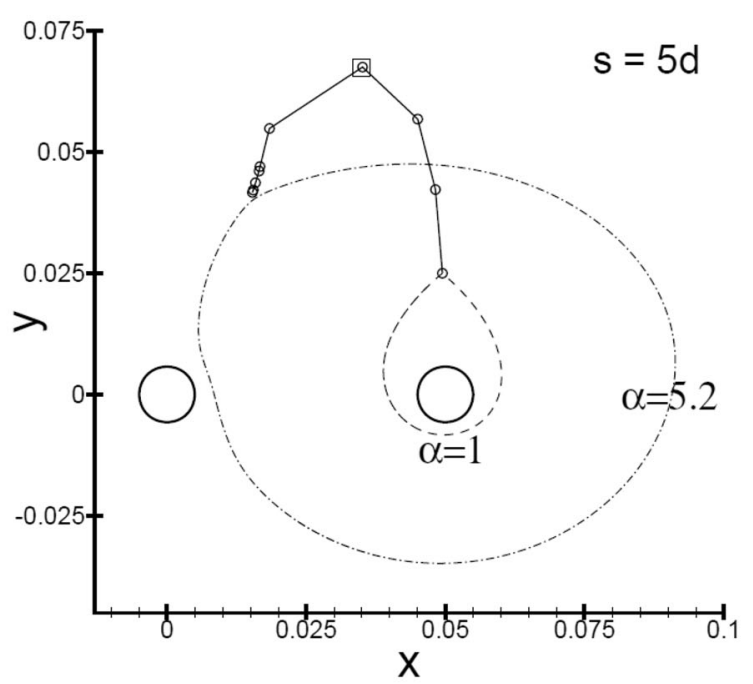

(c)

Figure 10. Locations of stagnation points with increase in rotational speed for inter-cylinder spacing values of (a) $2 \mathrm{~d}$, (b) $3.5 \mathrm{~d}$ and (c) $5 d$.

non-periodic shedding mentioned in section 5.2a. The mean drag force coefficient on upstream cylinder, i.e. $C_{d .1}$, initially increases with increase in $\alpha$, reaches a maximum value and then decreases. This maximum value is found to increase with increase in inter-cylinder spacing. The increase of $C_{d, 1}$ is found to coincide with the development of a stable circulation zone. The maximum value of $C_{d, 1}$ overlaps with $\alpha$ and s values at which shape change begins for circulation zone from droplet to elliptical. This transformation co-occurs with a decrease of drag on the upstream cylinder. It is also noted that the drag coefficient is lowest when $\alpha=5.2$ and $\mathrm{s}=3.5$.

Figure 11(b) presents the variation of mean drag coefficient on downstream cylinder $\left(C_{d, 2}\right)$ where multiple peaks suggest better coherence of mean drag with transition characteristics of downstream cylinder wake. The difference between maximum and minimum values of drag coefficient for $\alpha$ in the range $0 \leq \alpha \leq 1.2$ becomes quite significant for larger values of inter-cylinder spacing (3.5d and 5d). Strong proximity interference at small spacing could be the plausible cause for this behavior. However, with increase of inter-cylinder spacing, effect of proximity interference decreases and the rotational effect begins to dominate causing sudden variation in drag. For $\alpha=0$, the mean drag coefficient of the downstream cylinder is negative for all values of $s$, except for $s=5 d$, which confirms presence of a standing vortex in the space between cylinders. As the downstream cylinder begins to rotate, flow in the gap suffers deviation due to rotation. This flow impinges on the leading surface of the downstream cylinder creating a stagnation zone with increased pressure. In figure 11(c), the combined drag, defined as sum of drag on both cylinders, is plotted against non-dimensional rotational speed for different values of inter-cylinder spacing. The highlighted region in the figure corresponds to certain combinations of rotational speeds and values of intercylinder spacing for which overall drag becomes lower than that observed for the case of stationary tandem cylinders. 


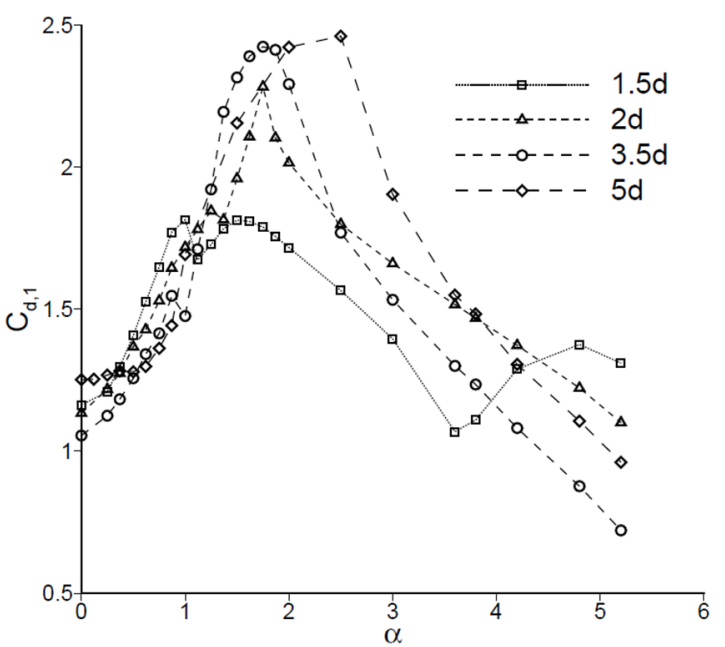

(a)

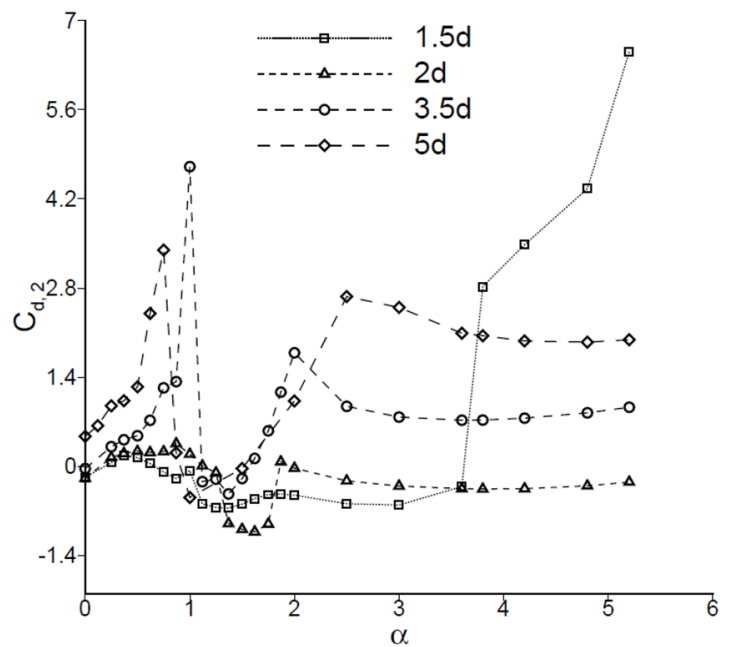

(b)

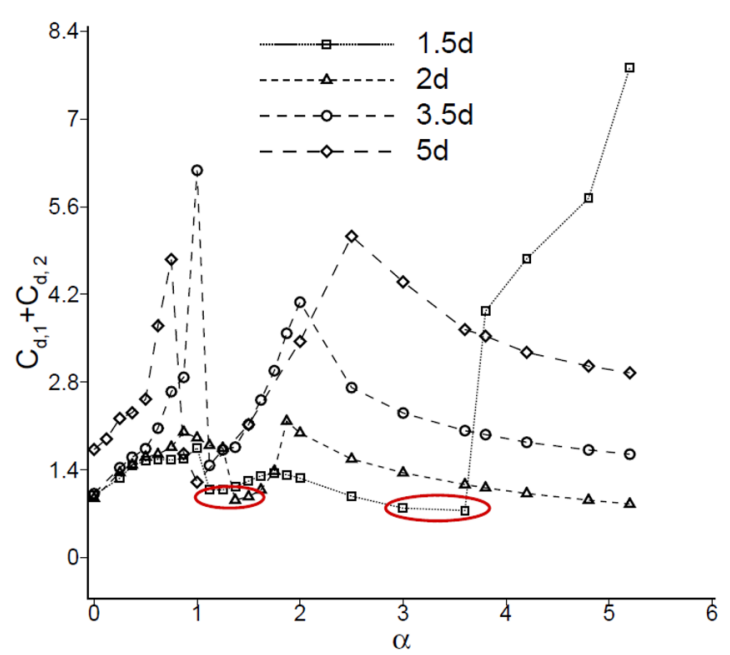

(c)

Figure 11. Variation of mean drag coefficient with rotational speed for different values of s, for (a) upstream cylinder, (b) downstream cylinder and (c) both cylinders.

This clearly illustrates promise of such combination of cylinder pair towards drag control.

The time-averaged pressure distribution on the surface of downstream cylinder in figure 12 clearly shows presence of the stagnation zone. Moreover, the low pressure region in wake of the downstream cylinder grows with increase in $\alpha$ and is the main cause for initial increase in drag coefficient of the cylinder. With further increase in $\alpha$, the drag coefficient attains its lowest value at a particular $\alpha$ that depends on value of $s$. This range of rotational speed coincides with formation and stabilization of circulation zone during which the flow evolves to a steady state from an unsteady one. At $\alpha$ values beyond this, the mean drag coefficient increases to attain a peak and then begins to decrease, the peak corresponding to change in shape of circulation zone as described in previous section.

5.4b Variation of lift: The resultant force acting on either cylinder in a direction perpendicular to the flow is normally expressed in terms of non-dimensionalized 'lift coefficient' $\left(C_{l}\right)$. The root mean square (RMS) value of the lift coefficient acting on upstream cylinder is found to increase with increase in $\alpha$ as shown in figure 13(a). This is because of change in angle of impingement of the flow on upstream cylinder due to ratation of downstream cylinder. As the angle of the impinging flow is increased due to increase of rotation, the vertical component of the resultant force gets enhanced. For $\alpha<1$, the value of $C_{l}$ for upstream cylinder does not differ significantly for other values of spacing except $\mathrm{s}=5 \mathrm{~d}$. This is due to vortex shedding from the upstream cylinder in the intercylinder space. In fact, for low rotational speeds, even change in $\alpha$ has least effect on variation of $C_{l}$. When $\alpha$ is increased further, a steep rise in lift coefficient is observed. This sudden change in value of $C_{l}$ may be attributed to development of circulation zone and continues till another transformation takes place in structure of the circulation zone. The location of stagnation zone 


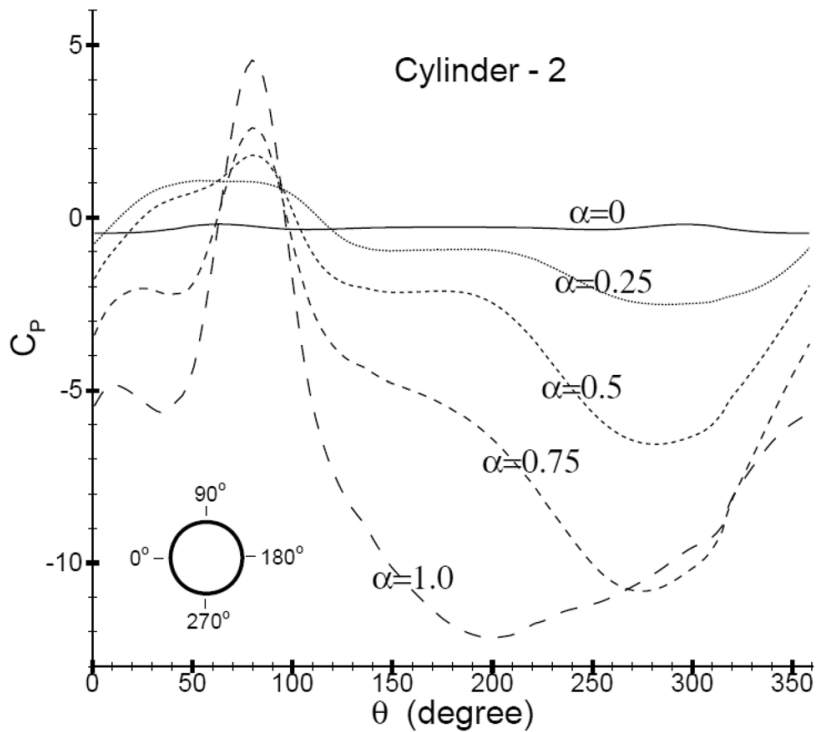

Figure 12. Time-averaged distribution of pressure coefficient on the surface of downstream cylinder at low rotational speeds $(\alpha<$ $1)$, for $s=3.5 \mathrm{~d}$.

corresponding to this transformation is highlighted in figure 10. Beyond this value of $\alpha$, change in lift coefficient of upstream cylinder with increase in $\alpha$ is rather small. Figure 13(b) depicts the variation of RMS lift coefficient on the downstream cylinder $\left(C_{1,2}\right)$ for different values of $\alpha$ and s. It is observed that $C_{1,2}$ increases with increase in rotational speed upto a particular value of $\alpha$ beyond which it shows decline and then becomes almost indipendent of $\alpha$. Figure 13(b) also shows that with increase in s, location of peak of $C_{1,2}$ shifts to larger values of $\alpha$ along with a large increase in magnitude of peak value itself. Once again, similar to the

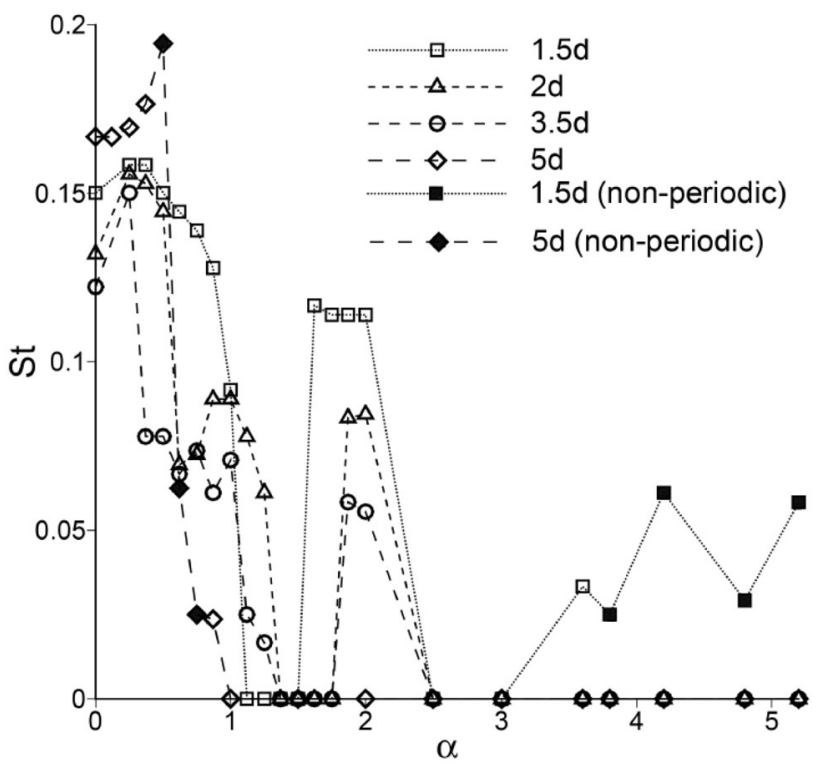

Figure 14. Variation of Strouhal number (St) with $\alpha$ for different values of inter-cylinder spacing (the darkened scatters correspond to non-periodic flow).

upstream cylinder, the maximum value of $C_{1,2}$ corresponds to transfornation in shape of the circulation zone.

\subsection{Temporal characteristics of the wake}

The temporal behavior of the flow field has been analyzed using time series of dynamic coefficients. The signals have been considered after 100 seconds of simulation to allow for stabilization of the flow and eliminate the initial transients. In order to investigate the periodic characteristics of

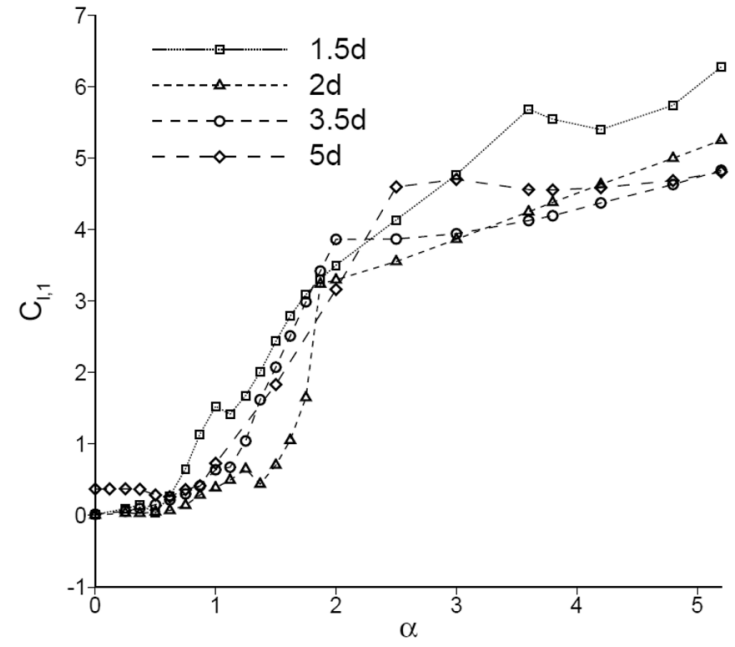

(a)

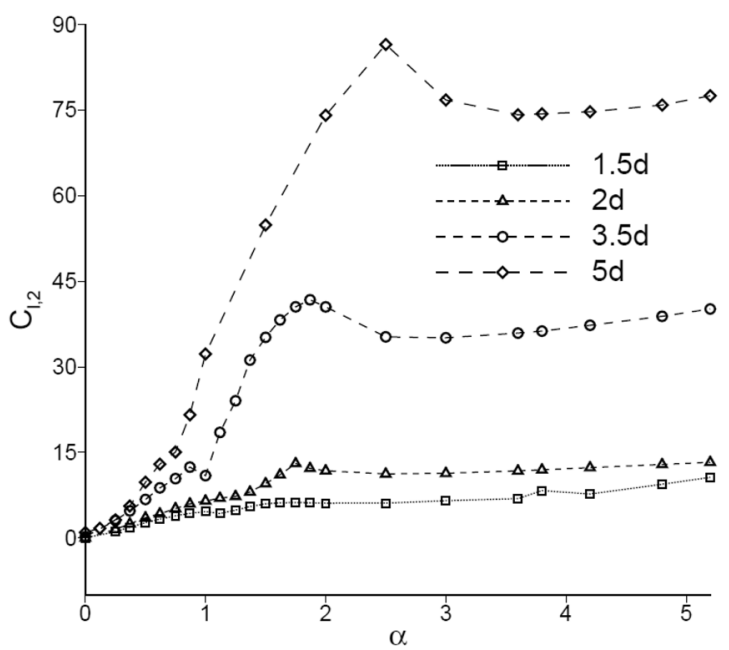

(b)

Figure 13. Variation of RMS lift coefficient with rotational speed for different values of inter-cylinder spacing on (a) upstream cylinder and (b) downstream cylinder. 

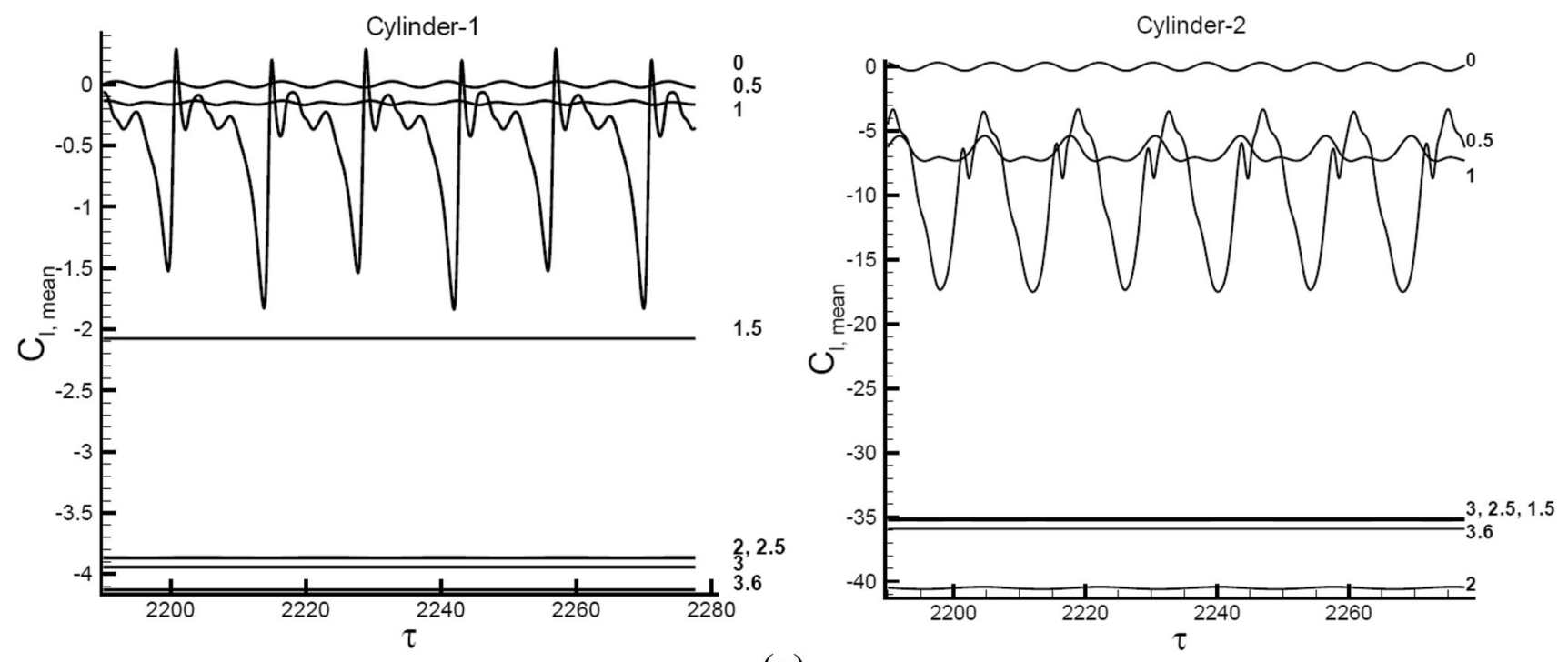

(a)

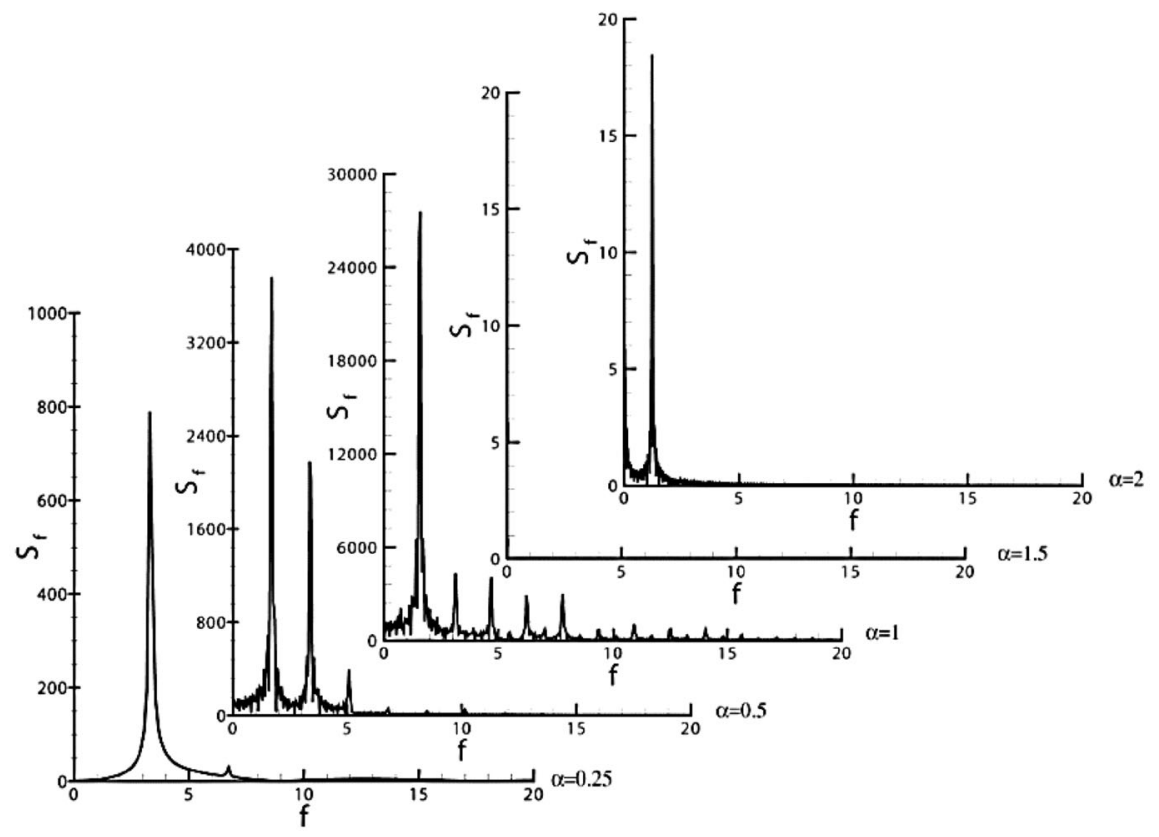

(b)

Figure 15. (a) Variation of lift on both the cylinders and (b) spectra of the lift signal of downstream cylinder, for $\mathrm{s}=3.5 \mathrm{~d}$.

signals, various derivatives such as Strouhal number, spectra and phase plots have been obtained. The Strouhal number has been defined based on the wake frequency $(f)$ of the downstream cylinder and is expressed as

$$
S t=\frac{f d}{U_{\infty}}
$$

Figure 14 shows the variation of St with $\alpha$ for different values of inter-cylinder spacing. For small and medium separation (1.5d, $2 \mathrm{~d}$ and $3.5 \mathrm{~d})$, two bands of $\alpha$ values, separated by a steady regime, have been identified to correspond to vortex shedding. However, the frequency of vortex shedding in the second band decreases with increase in spacing and vanishes for $\mathrm{s}=5 \mathrm{~d}$.

Various ranges of rotational speed and inter-cylinder spacing that correspond to suppression of vortex shedding $(S t=0)$ can be clearly identified in figure 14. Identification of such ranges of $\alpha$ and s becomes extremely important when the objective relates to flow control. There are two bands of $\alpha$ values that satisfy this criterion. The band corresponding to relatively larger rotational speed sustains undesirable large circulation zones that modify the free stream flow and 


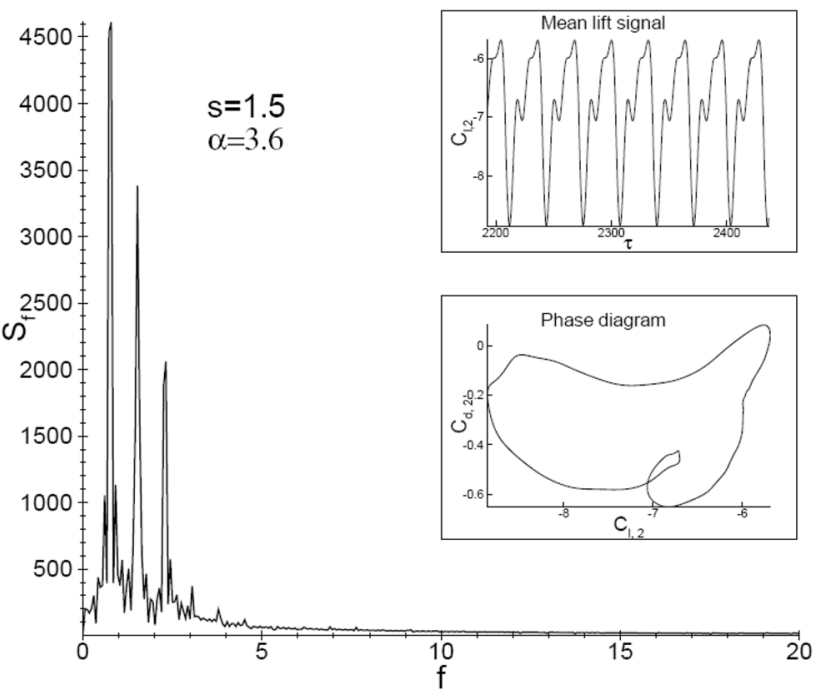

(a)

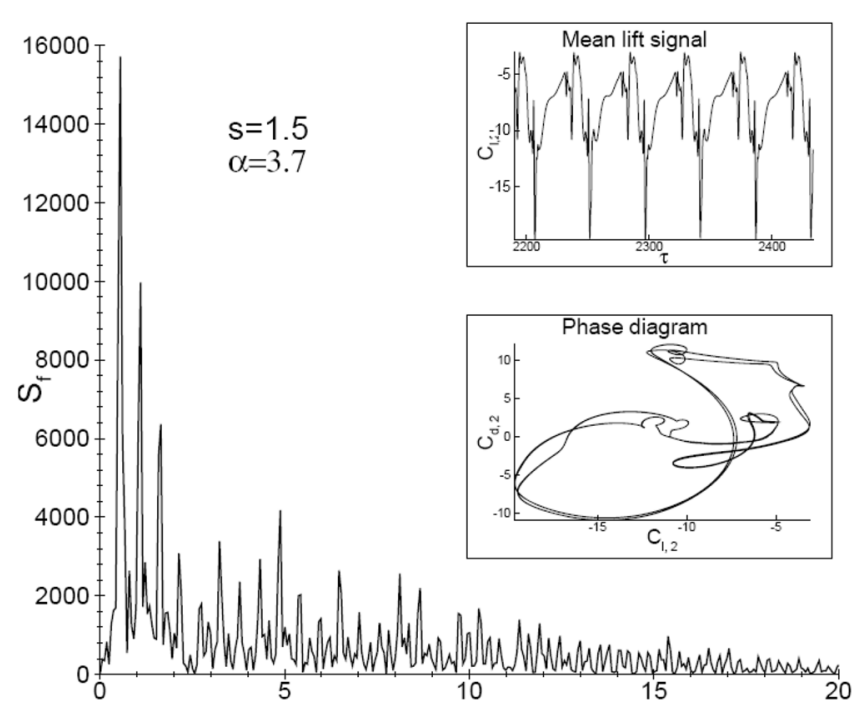

(b)

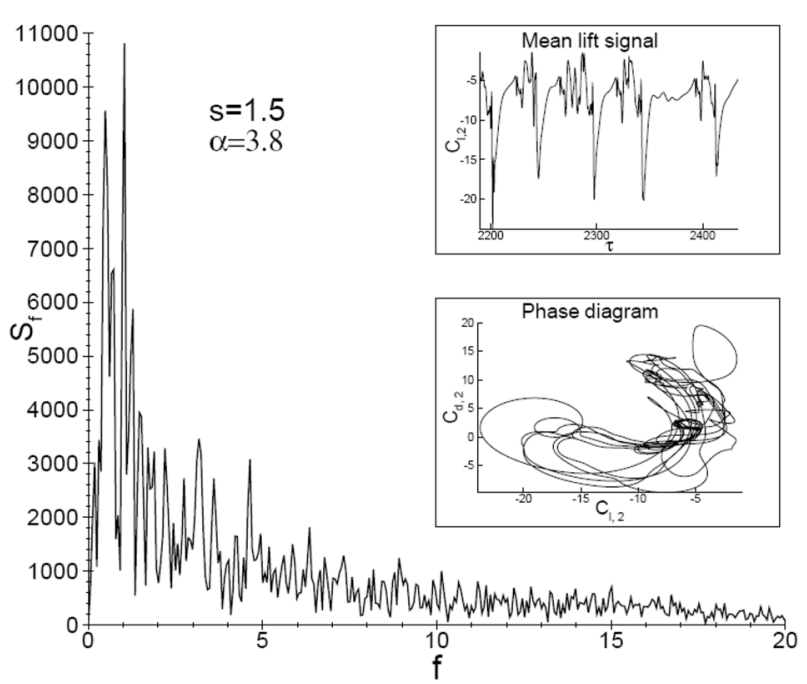

(c)

Figure 16. Temporal characterization of downstream cylinder wake for $\mathrm{s}=1.5 \mathrm{~d}$ (a) $\alpha=3.6$, (b) $\alpha=3.7$ and (c) $\alpha=3.8$.

generate significant aerodynamic forces. Accordingly, the band of $\alpha$ in the range from 1.0 to 2.0 is identified as the best choice for flow control. The width of this band is found to increase with increase in spacing between the cylinders. Since the size of circulation zone is small for small values of $\mathrm{s}$, a spacing of $\mathrm{s}=1.5 \mathrm{~d}$ and $\alpha$ in the range from 1.1 to 1.5 forms the best parametric combination towards flow control.

Vortex shedding during first unsteady range of $\alpha$ exhibits periodic, quasiperiodic and aperiodic nature depending on value of $s$ while shedding in the second unsteady band is always periodic. Change in periodic nature of the flow as it traverses through unsteady bands of $\alpha$ values have been presented in figure 15 for $\mathrm{s}=3.5 \mathrm{~d}$. It depicts the variation of lift coefficient on both the cylinders with respect to nondimensional time, $\left(\tau=\frac{U_{\infty} t}{d}\right)$ and the corresponding Fourier spectra. For small values of $\alpha$ in the range from 0 to 0.25 , presence of either single peak or multiple peaks that are superharmonics of dominant frequency, confirm the periodic nature of the flow. On further increase of $\alpha$, spectral distributions show multiple peaks corresponding to frequency values that do not remain integer multiples of each other. This flow regime has been categorized as 'quasiperiodic' and appears in the range $0.25 \leq \alpha \leq 1$.

In figure 14 , signal for $\alpha=1.5$ indicates that the flow is steady and actually falls within a band of $\alpha$ corresponding to steady flow regime. The second band of vortex shedding that illustrates periodic nature begins close to $\alpha=1.8$ and continues till $\alpha$ value becomes slightly less than 2.5 . The reason for re-onset of vortex shedding in this range may be attributed to asymmetry in the wake of upstream cylinder as 
seen from the vorticity contour of figure 7 , say for $\alpha=2$. For the case of $\mathrm{s}=3.5 \mathrm{~d}$, the flow again becomes steady and continues to be so with further increase in value of $\alpha$. The $\alpha$ values that correspond to onset of re-stabilization of the flow are almost same for all values of s. However, for $\mathrm{s}=1.5 \mathrm{~d}$, the flow does not continue to remain stable and transits to an aperiodic regime beyond $\alpha=3$. The values of $\alpha$ that correspond to aperiodic shedding have been highlighted using solid markers in figure 14. It has been noted such aperiodic shedding is observed only for spacing values of $1.5 \mathrm{~d}$ and $5 \mathrm{~d}$.

Figure 16 presents the transitional characteristics of the wake from periodic to aperiodic shedding with the help of phase plot, time signal and its spectra, for increasing values of $\alpha$ and fixed value of $\mathrm{s}=1.5 \mathrm{~d}$. The phase plot considers a mutual dependence of $C_{l}$ and $C_{d}$ contrasting their time variation. For $\alpha=3.6$, the power spectrum shows three dominant peaks with frequencies in the ratio 1:2.3 which means the flow is periodic. However, slight deviation in the signal from its pure sinusoidal nature and similar deviation in the phase diagram, indicate the presence of weak secondary frequencies. As $\alpha$ increased to 0.37 , the secondary frequencies become enough significant and the phase path shows two loops. This regime is defined as 'quasiperiodic'. With further increase in $\alpha$, say for $\alpha=3.8$ and beyond, the flow seems to demonstrate aperiodic behavior. The aperiodicity is characterized by the presence of a continuous band in the power spectrum and multi-loops in the phase diagram. Similar transitions towards aperiodicity have been observed for $\mathrm{s}=5 \mathrm{~d}$ in $\alpha$ range $0.6-0.8$. Beyond this value of $\alpha$, vortex shedding again becomes periodic and finally gets arrested around $\alpha=1$.

\section{Conclusion}

Flow past two inline circular cylinders with rotating downstream cylinder has been studied at $\mathrm{Re}=150$ to bring out the effects of rotational motion and inter-cylinder spacing on wake and dynamic characteristics. The non-dimensional rotational speed is varied between 0 and 5.2 for inter-cylinder spacing values of $1.5 \mathrm{~d}, 2 \mathrm{~d}, 3.5 \mathrm{~d}$ and $5 \mathrm{~d}$. The flow characteristics reveal different modes of vortex shedding, multiple transitions in temporal response of the wake and structural transformation of the circulation zone. The circulation zone is found to be a critical flow feature that alters both temporal and dynamic characteristics. The dynamic characteristics reveal that significant drag reduction is possible for upstream cylinder in certain range of values of rotational speed of downstream cylinder $(\alpha)$ and spacing between the cylinders (s). The signals of lift and drag, their power spectra and phase diagram closely illustrate transition of the downstream cylinder wake to an aperiodic regime through periodic and quasiperiodic routes. Such transitions are only found to be present for $\mathrm{s}=1.5 \mathrm{~d}$ and $\mathrm{s}=5 \mathrm{~d}$. Moreover, optimal bands of $\alpha$ values have been obtained corresponding to each value of s that are associated with suppression of vortex shedding. Suppression of vortex shedding at large values of $\alpha$ is found to be closely associated with development of a stable circulation zone. Results from the present work promise us to use of such an arrangement for engineering applications involving drag reduction and flow control. However, it is noted that further studies that disclose the influence of size of rotating control rod and $\mathrm{Re}$ on development of circulation zone are to be undertaken in order to identify a general spectrum of parameters for flow control applications.

\section{References}

[1] Ingham D B 1983 Steady flow past a rotating cylinder. Comput. Fluids 11(4): 351-366

[2] Tang T and Ingham D B 1991 On steady flow past a rotating circular cylinder at Reynolds numbers 60 and 100. Comput. Fluids 19(2): 217-230

[3] Badr H M, Dennis S C R and Young P J S 1989 Steady and unsteady flow past a rotating circular cylinder at low Reynolds numbers. Comput. Fluids 17(4): 579-609

[4] Kang S, Choi H and Lee S 1999 Laminar flow past a rotating circular cylinder. Phys. Fluids 11(11): 3312-3321

[5] Manoj N, Tapan K Sengupta and Umendra S Chauhan 1998 Flow past rotating cylinders at high Reynolds numbers using higher order upwind scheme. Comput. Fluids 27(1): 47-70

[6] Mittal S and Kumar B 2003 Flow past a rotating cylinder. $J$. Fluid Mech. 476: 303-334

[7] Kumar S, Cantu C and Gonzalez B 2011a Flow past a rotating cylinder at low and high rotation rates. J. Fluids Eng. 133(4): 041201

[8] Meena J and Mittal S 2015 Three-dimensional flow past a rotating cylinder. J. Fluid Mech. 766: 28-53

[9] Rao A, Radi A, Leontini J S and Thompson M C 2015 A review of rotating cylinder wake transitions. J. Fluids Struct. 53: 2-14

[10] Carmo B S and Meneghini J R 2006 Numerical investigation of the flow around two circular cylinders in tandem. J. Fluids Struct. 22(6): 979-988

[11] Chan A S, Dewey P A, Jameson A, Liang C and Smits A J 2011 Vortex suppression and drag reduction in the wake of counter-rotating cylinders. J. Fluid Mech. 679: 343-382

[12] Chan A S and Jameson A 2010 Suppression of the unsteady vortex wakes of a circular cylinder pair by a doublet-like counter-rotation. Int. J. Numer. Methods Fluids 63(1): 22-39

[13] Guo X, Lin J, Cheng-xu T U and Wang H 2009 Flow past two rotating circular cylinders in a side-by-side arrangement. J. Hydrodyn. Ser. B 21(2): 143-151

[14] Kumar S, Gonzalez B and Probst O 2011b Flow past two rotating cylinders. Phys. Fluids 23(1): 014102

[15] Yoon H S, Kim J H, Chun H H, Choi H J 2007 Laminar flow past two rotating circular cylinders in a side-by-side arrangement. Phys. Fluids 19(12): 128103

[16] Shaafi K and Vengadesan S 2014 Wall proximity effects on the effectiveness of upstream control rod. J. Fluids Struct. 49: 112-134 
[17] Ayyappan T and Vengadesan S 2008 Influence of staggering angle of a rotating rod on flow past a circular cylinder. $J$. Fluids Eng. 130(3): 031103

[18] Nemati H, Farhadi M, K Sedighi, M M Pirouz and Arabati N N 2012 Convective heat transfer from two rotating circular cylinders in tandem arrangement by using lattice Boltzmann method. Appl. Math. Mech. 33(4): 427-444

[19] Mittal S 2001 Control of flow past bluff bodies using rotating control cylinders. J. Fluids Struct. 15(2): 291-326

[20] Mittal S and Raghuvanshi A 2001 Control of vortex shedding behind circular cylinder for flows at low Reynolds numbers. Int. J. Numer. Methods Fluids 35(4): 421-447
[21] Meneghini J R, Saltara F, Siquera C L R and Ferrari J A 2001 Numerical simulation of flow interference between two circular cylinders in tandem and side-by-side arrangements. $J$. Fluids Struct. 15(2): 327-350

[22] Slaouti A and Stansby P K1992 Flow around two circular cylinders by the random-vortex method. J. Fluids Struct. 6: 641-670

[23] Igarashi T 1981 Characteristics of the flow around two circular cylinders arranged in tandem. Bull. JSME 24.188: 323-331

[24] Williamson and Roshko 1988 Vortex formation in the wake of an oscillatory cylinder. J. Fluids Struct. 28(2): 355-381

[25] Jeang J and Hussain F 1995 On identification of a vortex. $J$. Fluid Mech. 285: 69-94 